\title{
Existence of ground state solutions for a class of Choquard equations with local nonlinear perturbation and variable potential
}

Jing Zhang ${ }^{1,2}$ and Qiongfen Zhang ${ }^{1,2^{*}}$ (1)

\section{"Correspondence:}

qfzhangcsu@163.com

${ }^{1}$ College of Science, Guilin

University of Technology, Guilin,

Guangxi 541004, P.R. China

${ }^{2}$ Guangxi Colleges and Universities

Key Laboratory of Applied Statistics,

Guilin, Guangxi 541004, P.R. China

\section{Springer}

\begin{abstract}
In this paper, we focus on the existence of solutions for the Choquard equation

$$
\left\{\begin{array}{l}
-\Delta u+V(x) u=\left(I_{\alpha} *|u|^{\frac{\alpha}{N}+1}\right)|u|^{\frac{\alpha}{N}-1} u+\lambda|u|^{p-2} u, \quad x \in \mathbb{R}^{N} ; \\
u \in H^{1}\left(\mathbb{R}^{N}\right),
\end{array}\right.
$$

where $\lambda>0$ is a parameter, $\alpha \in(0, N), N \geq 3, I_{\alpha}: \mathbb{R}^{N} \rightarrow \mathbb{R}$ is the Riesz potential. As usual, $\alpha / N+1$ is the lower critical exponent in the Hardy-Littlewood-Sobolev inequality. Under some weak assumptions, by using minimax methods and Pohožaev identity, we prove that this problem admits a ground state solution if $\lambda>\lambda_{*}$ for some given number $\lambda_{*}$ in three cases: (i) $2<p<\frac{4}{N}+2$, (ii) $p=\frac{4}{N}+2$, and (iii) $\frac{4}{N}+2<p<2^{*}$. Our result improves the previous related ones in the literature.
\end{abstract}

MSC: 34 C $37 ; 35 \mathrm{~A} 15 ; 37 J 45 ; 47 J 30$

Keywords: Ground state solution; Variable potential; Choquard equation; Critical point

\section{Introduction}

In this paper, we mainly study the Choquard equation with a variable potential and a local nonlinearity:

$$
\left\{\begin{array}{l}
-\Delta u+V(x) u=\left(I_{\alpha} *|u|^{\frac{\alpha}{N}+1}\right)|u|^{\frac{\alpha}{N}-1} u+\lambda|u|^{p-2} u, \quad x \in \mathbb{R}^{N} \\
u \in H^{1}\left(\mathbb{R}^{N}\right)
\end{array}\right.
$$

where $\lambda>0, \alpha \in(0, N), N \geq 3,2<p<2^{*}$, and $I_{\alpha}: \mathbb{R}^{N} \rightarrow \mathbb{R}$ is the Riesz potential defined by

$$
I_{\alpha}(x)=\frac{\Gamma\left(\frac{N-\alpha}{2}\right)}{\Gamma\left(\frac{\alpha}{2}\right) 2^{\alpha} \pi^{N / 2}|x|^{N-\alpha}}, \quad x \in \mathbb{R}^{N} \backslash\{0\} .
$$

$V: \mathbb{R}^{N} \rightarrow \mathbb{R}$ satisfies the following assumptions:

(c) The Author(s) 2021. This article is licensed under a Creative Commons Attribution 4.0 International License, which permits use, sharing, adaptation, distribution and reproduction in any medium or format, as long as you give appropriate credit to the original author(s) and the source, provide a link to the Creative Commons licence, and indicate if changes were made. The images or other third party material in this article are included in the article's Creative Commons licence, unless indicated otherwise in a credit line to the material. If material is not included in the article's Creative Commons licence and your intended use is not permitted by statutory regulation or exceeds the permitted use, you will need to obtain permission directly from the copyright holder. To view a copy of this licence, visit http://creativecommons.org/licenses/by/4.0/. 
(V1) $V \in \mathcal{C}\left(\mathbb{R}^{N},[0, \infty)\right)$;

(V2) $V(x) \leq V_{\infty}:=\lim _{|y| \rightarrow \infty} V(y)<\infty$ for all $x \in \mathbb{R}^{N}$.

By the Hardy-Littlewood-Sobolev inequality (see Lemma 2.1), one has

$$
\left[\int_{\mathbb{R}^{N}}\left(I_{\alpha} *|u|^{\frac{\alpha}{N}+1}\right)|u|^{\frac{\alpha}{N}+1} d x\right]^{\frac{N}{N+\alpha}} \leq S^{-1} \int_{\mathbb{R}^{N}} u^{2} d x .
$$

In the work of Lieb and Loss (see [1]), the sharp constant $S$ is achieved by a function $u \in H^{1}\left(\mathbb{R}^{N}\right)$ if and only if, for every $x \in \mathbb{R}^{N}$,

$$
u(x)=A\left(z^{2}+|x-a|^{2}\right)^{-\frac{N}{2}}
$$

for $a \in \mathbb{R}^{N}, A>0$, and $z>0$. In Lemma 2.9, we choose $A=A_{0}>0$, and $A_{0}$ is determined by

$$
A_{0}^{\frac{2 \alpha}{N}+2} \int_{\mathbb{R}^{N}} \int_{\mathbb{R}^{N}} \frac{d x d z}{\left(1+|x|^{2}\right)^{\frac{N+\alpha}{2}}|x-z|^{N-\alpha}\left(1+|z|^{2}\right)^{\frac{N+2}{2}}}=\frac{2^{\alpha} \pi^{\frac{N}{2}} \Gamma\left(\frac{\alpha}{2}\right)}{\Gamma\left(\frac{N-\alpha}{2}\right)} .
$$

Under (V1), (V2), (1.2), and the Sobolev embedding theorem, the weak solutions of (1.1) correspond to the critical points of the energy functional $\mathcal{I}: H^{1}\left(\mathbb{R}^{N}\right) \rightarrow \mathbb{R}$ defined by

$$
\begin{aligned}
\mathcal{I}(u)= & \frac{1}{2} \int_{\mathbb{R}^{N}}\left[|\nabla u|^{2}+V(x) u^{2}\right] d x-\frac{N}{2(N+\alpha)} \int_{\mathbb{R}^{N}}\left(I_{\alpha} *|u|^{\frac{\alpha}{N}+1}\right)|u|^{\frac{\alpha}{N}+1} d x \\
& -\frac{\lambda}{p} \int_{\mathbb{R}^{N}}|u|^{p} d x,
\end{aligned}
$$

which is continuously differentiable and

$$
\begin{aligned}
\left\langle\mathcal{I}^{\prime}(u), v\right\rangle= & \int_{\mathbb{R}^{N}}(\nabla u \cdot \nabla v+V(x) u v) d x-\int_{\mathbb{R}^{N}}\left(I_{\alpha} *|u|^{\frac{\alpha}{N}+1}\right)|u|^{\frac{\alpha}{N}-1} u v d x \\
& -\lambda \int_{\mathbb{R}^{N}}|u|^{p-2} u v d x, \quad \forall v \in H^{1}\left(\mathbb{R}^{N}\right) .
\end{aligned}
$$

If the potential $V(x) \equiv V_{\infty}$, then (1.1) reduces to the autonomous equation

$$
\left\{\begin{array}{l}
-\Delta u+V_{\infty} u=\left(I_{\alpha} *|u|^{\frac{\alpha}{N}+1}\right)|u|^{\frac{\alpha}{N}-1} u+\lambda|u|^{p-2} u, \quad x \in \mathbb{R}^{N} ; \\
u \in H^{1}\left(\mathbb{R}^{N}\right) .
\end{array}\right.
$$

Similar to (1.5), the energy functional of (1.7) is defined by

$$
\begin{aligned}
\mathcal{I}^{\infty}(u)= & \frac{1}{2} \int_{\mathbb{R}^{N}}\left[|\nabla u|^{2}+V_{\infty} u^{2}\right] d x-\frac{N}{2(N+\alpha)} \int_{\mathbb{R}^{N}}\left(I_{\alpha} *|u|^{\frac{\alpha}{N}+1}\right)|u|^{N^{\alpha}+1} d x \\
& -\frac{\lambda}{p} \int_{\mathbb{R}^{N}}|u|^{p} d x .
\end{aligned}
$$

Equation (1.1) is a special form of the following Choquard equation with a local nonlinear perturbation and a variable potential:

$$
\left\{\begin{array}{l}
-\Delta u+V(x) u=\left(I_{\alpha} *|u|^{q}\right)|u|^{q-2} u+f(u), \quad x \in \mathbb{R}^{N} \\
u \in H^{1}\left(\mathbb{R}^{N}\right)
\end{array}\right.
$$

where $1+\frac{\alpha}{N}<q<\frac{N+\alpha}{N-2}$. 
If $f=0$ and $V(x) \equiv 1,(1.9)$ appears under the background of various physical models. For example, as early as in 1954, Pekar [2] introduced (1.9) into the physical model to study the free electrons in a ionic lattice interact with phonons associated with deformations of the lattice. Choquard equation is also known as the Schrödinger-Newton equation after the addition of non-relativistic Newtonian gravity to some Schrödinger equations [3-6]. Lieb [7] first verified the positive solution of (1.9) in $\mathbb{R}^{3}$ when $f=0, \alpha=2, V(x) \equiv 1$, and $q=2$. Later, Lions $[8,9]$ further improved the results of (1.9) and obtained the existence and multiplicity of normalized solution for (1.9). The existence of a ground state solution and the qualitative properties of the solution in the range of exponents $q$ which satisfies

$$
1+\frac{\alpha}{N}<q<\frac{N+\alpha}{N-2}
$$

were established in [10].

The endpoints $\frac{N+\alpha}{N-2}$ and $\frac{N+\alpha}{N}$ are critical exponents. It is known to all that $\frac{N+\alpha}{N-2}$ is an upper critical exponent which plays a similar role as the Sobolev critical exponent in the local semilinear equations [11-17]. The lower critical exponent $\frac{N+\alpha}{N}$ is strictly greater than 1 which comes from inequality (1.2). So far, many authors have investigated the existence of nontrivial solutions of many forms of (1.9) (see [18-21]). In addition, for some applications of the variational method in elliptic systems, we refer to [22-24]. If the potential $V(x) \equiv 1$, then (1.1) reduces to the following equation:

$$
\left\{\begin{array}{l}
-\Delta u+u=\left(I_{\alpha} *|u|^{\frac{\alpha}{N}+1}\right)|u|^{\frac{\alpha}{N}-1} u+\lambda|u|^{p-2} u, \quad x \in \mathbb{R}^{N} \\
u \in H^{1}\left(\mathbb{R}^{N}\right) .
\end{array}\right.
$$

Tang, Wei, and Chen [25] proved that (1.10) has ground state solutions in the following assumptions:

(i) $2<p<\frac{4}{N}+2$ and $\lambda>0$;

(ii) $p=\frac{4}{N}+2$ and $\lambda>\frac{N^{2}}{A_{0}^{\frac{4}{N}} S^{\frac{2}{\alpha}}}$;

(iii) $\frac{4}{N}+2<p<2^{*}$ and $\lambda>\frac{p N^{4} \Gamma \frac{p N}{2} \Gamma \frac{N}{2}}{8(N+1) !\left(A_{0} t_{0}\right)^{p-2} \Gamma \frac{(p-1) N}{2}}$.

By using the mountain pass lemma, they obtained a Palais-Smale sequence and the corresponding energy level $m$. Then, from these three assumptions, an estimate of the energy level $m$ was given, which is very important to ensure the Sobolev compactness. We further improve these three hypotheses to be applicable to the research in this paper. This has certain enlightenment to our work.

Motivated by the work of $[26,27]$, we use a weaker decay assumption on $\nabla \mathrm{V}$ to solve the trouble caused by variable potential.

(V3) $V \in \mathcal{C}^{1}\left(\mathbb{R}^{N}, \mathbb{R}\right)$, and there is $\theta \in[0,1)$ such that

$$
\nabla V(x) \cdot x \leq \frac{(N-2)^{2} \theta}{2|x|^{2}}, \quad \forall x \in \mathbb{R}^{N} \backslash\{0\}
$$

Van Schaftingen and Xia [11], Chen and Tang [26] did a pretty good job, which gives us some inspiration. To our knowledge, there seems to be no results of (1.1). Motivated by the above works, especially $[25,26]$, in this paper, we establish the existence result of 
ground state solutions for (1.1). To state our result, inspired by [28], we define the following Pohožaev identity functional on $H^{1}\left(\mathbb{R}^{N}\right)$ :

$$
\begin{aligned}
\mathcal{P}(u):= & \frac{N-2}{2}\|\nabla u\|_{2}^{2}+\frac{1}{2} \int_{\mathbb{R}^{N}}[N V(x)+\nabla V(x) \cdot x] u^{2} d x \\
& -\frac{N}{2} \int_{\mathbb{R}^{N}}\left(I_{\alpha} *|u|^{\frac{\alpha}{N}+1}\right)|u|^{\frac{\alpha}{N}+1} d x-\frac{N \lambda}{p} \int_{\mathbb{R}^{N}}|u|^{p} d x,
\end{aligned}
$$

and

$$
\begin{aligned}
\mathcal{P}^{\infty}(u):= & \frac{N-2}{2}\|\nabla u\|_{2}^{2}+\frac{N V_{\infty}}{2}\|u\|_{2}^{2}-\frac{N}{2} \int_{\mathbb{R}^{N}}\left(I_{\alpha} *|u|^{\frac{\alpha}{N}+1}\right)|u|^{\frac{\alpha}{N}+1} d x \\
& -\frac{N \lambda}{p} \int_{\mathbb{R}^{N}}|u|^{p} d x .
\end{aligned}
$$

In view of [29, Prorosition 3.1], if $\bar{u}$ is a solution of (1.1), then it satisfies the Pohožaev identity $\mathcal{P}(u)=0$. Let

$$
\mathcal{M}:=\left\{u \in H^{1}\left(\mathbb{R}^{N}\right) \backslash\{0\}: \mathcal{P}(u)=0\right\} .
$$

Our main result is as follows.

Theorem 1.1 Assume that $V$ satisfies $(V 1)-(V 3)$ and one of the following conditions:

(i) $2<p<\frac{4}{N}+2$ and $\lambda>0$;

(ii) $p=\frac{4}{N}+2$ and $\lambda>\frac{(N+2) N^{2}}{2(N+1) A_{0}^{\frac{4}{N}}\left(S V_{\infty}\right)^{\frac{2}{\alpha}}}$;

(iii) $\frac{4}{N}+2<p<2^{*}$ and $\lambda>\frac{25 p N^{4} \Gamma\left(\frac{N}{2}\right) \Gamma\left(\frac{p N}{2}\right)}{256(N+1) ! A_{0}^{p-2} \varepsilon^{2 *-2}\left(V_{\infty} S\right)^{\frac{2}{\alpha}} \Gamma\left(\frac{(p-1) N}{2}\right)}$ holds. Then problem (1.1) has a solution $\bar{u} \in H^{1}\left(\mathbb{R}^{N}\right)$ such that

$$
\mathcal{I}(\bar{u})=\inf _{\mathcal{M}} \mathcal{I}=\inf _{u \in H^{1}\left(\mathbb{R}^{N}\right) \backslash\{0\}} \max _{t>0} \mathcal{I}\left(u_{t}\right)>0
$$

where $u_{t}(x):=u(x / t)$.

In this paper, we use the following notations:

- $H^{1}\left(\mathbb{R}^{N}\right)$ denotes the usual Sobolev space equipped with the inner product and the norm

$$
(u, v)=\int_{\mathbb{R}^{N}}(\nabla u \cdot \nabla v+u v) d x, \quad\|u\|=(u, u)^{1 / 2}, \quad \forall u, v \in H^{1}\left(\mathbb{R}^{N}\right) .
$$

- $L^{s}\left(\mathbb{R}^{N}\right)(1<s<\infty)$ denotes the Lebesgue space with the norm $\|u\|_{s}=\left(\int_{\mathbb{R}^{N}}|u|^{s} d x\right)^{1 / s}$.

- For any $u \in H^{1}\left(\mathbb{R}^{N}\right)$ and $r>0, B_{r}(x):=\{y \in \mathbb{R}:|y-x|<r\}$.

- For any $u \in H^{1}\left(\mathbb{R}^{N}\right) \backslash\{0\}, u_{t}(x):=u(x / t)$ for $t>0$.

- $C, C_{1}, C_{2}, \ldots$ denote positive constants possibly different in different places.

\section{Proof of the main result}

Before proving the main result, we first give some key inequalities and lemmas. The following famous Hardy-Littlewood-Sobolev inequality [1, Theorem 4.3] is an origin of the variational approach to (1.1). 
Lemma 2.1 Let $\alpha \in(0, N)$ and $s \in\left(1, \frac{N}{\alpha}\right)$. If $u \in L^{s}\left(\mathbb{R}^{N}\right)$, then $I_{\alpha} * u \in L^{\frac{N s}{N-\alpha s}}\left(\mathbb{R}^{N}\right)$, and

$$
\int_{\mathbb{R}^{N}}\left|I_{\alpha} * u\right|^{\frac{N s}{N-\alpha s}} d x \leq C\left(\int_{\mathbb{R}^{N}}|u|^{s} d x\right)^{\frac{N}{N-\alpha s}}
$$

where the constant $C>0$ depends only on $\alpha, N$, and $s$.

By a simple calculation, we have the following lemma.

Lemma 2.2 The following two inequalities hold:

$$
\begin{aligned}
& g(t):=2-N t^{N-2}+(N-2) t^{N} \geq 0, \quad \forall t \in[0,+\infty), \\
& \beta(t):=\alpha-(N+\alpha) t^{N}+N t^{N+\alpha} \geq \beta(1)=0, \quad \forall t \in[0,+\infty) .
\end{aligned}
$$

Moreover, (V3) implies that the following inequality holds:

$$
\begin{aligned}
& N t^{N}[V(x)-V(t x)]+\left(t^{N}-1\right) \nabla V(x) \cdot x \\
& \quad \geq-\frac{(N-2)^{2} \theta\left[2-N t^{N-2}+(N-2) t^{N}\right]}{4|x|^{2}}, \quad \forall t>0, x \in \mathbb{R}^{N} \backslash\{0\} .
\end{aligned}
$$

Lemma 2.3 Assume that (V1) and (V3) hold. Then

$$
\begin{aligned}
\mathcal{I}(u) \geq & \mathcal{I}\left(u_{t}\right)+\frac{1-t^{N}}{N} \mathcal{P}(u)+\frac{(1-\theta) g(t)}{2 N}\|\nabla u\|_{2}^{2} \\
& +\frac{\beta(t)}{2(N+\alpha)} \int_{\mathbb{R}^{N}}\left(I_{\alpha} *|u|^{\frac{\alpha}{N}+1}\right)|u|^{\frac{\alpha}{N}+1} d x, \quad \forall u \in H^{1}\left(\mathbb{R}^{N}\right), t>0 .
\end{aligned}
$$

Proof According to Hardy's inequality, we obtain

$$
\|\nabla u\|_{2}^{2} \geq \frac{(N-2)^{2}}{4} \int_{\mathbb{R}^{N}} \frac{u^{2}}{|x|^{2}} d x, \quad \forall u \in H^{1}\left(\mathbb{R}^{N}\right) .
$$

Note that

$$
\begin{aligned}
\mathcal{I}\left(u_{t}\right)= & \frac{t^{N-2}}{2}\|\nabla u\|_{2}^{2}+\frac{t^{N}}{2} \int_{\mathbb{R}^{N}} V(t x) u^{2} d x-\frac{t^{N} \lambda}{p} \int_{\mathbb{R}^{N}}|u|^{p} d x \\
& -\frac{N t^{N+\alpha}}{2(N+\alpha)} \int_{\mathbb{R}^{N}}\left(I_{\alpha} *|u|^{\frac{\alpha}{N}+1}\right)|u|^{\frac{\alpha}{N}+1} d x .
\end{aligned}
$$

Thus, by (1.5), (1.11), (2.2), (2.3), (2.4), (2.6), and (V3), one has

$$
\begin{aligned}
\mathcal{I}(u)-\mathcal{I}\left(u_{t}\right)= & \frac{1-t^{N-2}}{2}\|\nabla u\|_{2}^{2}+\frac{1}{2} \int_{\mathbb{R}^{N}}\left[V(x)-t^{N} V(t x)\right] u^{2} d x \\
& +\frac{N t^{N+\alpha}-N}{2(N+\alpha)} \int_{\mathbb{R}^{N}}\left(I_{\alpha} *|u|^{\frac{\alpha}{N}+1}\right)|u|^{\frac{\alpha}{N}+1} d x+\frac{\left(t^{N}-1\right) \lambda}{p} \int_{\mathbb{R}^{N}}|u|^{p} d x \\
= & \frac{1-t^{N}}{N}\left\{\frac{N-2}{2}\|\nabla u\|_{2}^{2}+\frac{1}{2} \int_{\mathbb{R}^{N}}[N V(x)+\nabla V(x) \cdot x] u^{2} d x\right.
\end{aligned}
$$




$$
\begin{aligned}
& \left.-\frac{N}{2} \int_{\mathbb{R}^{N}}\left(I_{\alpha} *|u|^{\frac{\alpha}{N}+1}\right)|u|^{\frac{\alpha}{N}+1} d x-\frac{N \lambda}{p} \int_{\mathbb{R}^{N}}|u|^{p} d x\right\}+\frac{g(t)}{2 N}\|\nabla u\|_{2}^{2} \\
& +\frac{1}{2} \int_{\mathbb{R}^{N}}\left\{t^{N}[V(x)-V(t x)]-\frac{1-t^{N}}{N} \nabla V(x) \cdot x\right\} u^{2} d x \\
& +\frac{\beta(t)}{2(N+\alpha)} \int_{\mathbb{R}^{N}}\left(I_{\alpha} *|u|^{\frac{\alpha}{N}+1}\right)|u|^{\frac{\alpha}{N}+1} d x \\
& \geq \frac{1-t^{N}}{N} \mathcal{P}(u)+\frac{(1-\theta) g(t)}{2 N}\|\nabla u\|_{2}^{2} \\
& +\frac{\beta(t)}{2(N+\alpha)} \int_{\mathbb{R}^{N}}\left(I_{\alpha} *|u|^{\frac{\alpha}{N}+1}\right)|u|^{\frac{\alpha}{N}+1} d x .
\end{aligned}
$$

From Lemma 2.3, we have the following corollary.

Corollary 2.4 Assume that (V1) and (V3) hold. Then, for $u \in \mathcal{M}$,

$$
\mathcal{I}(u)=\max _{t>0} \mathcal{I}\left(u_{t}\right)
$$

Based on the above results, we establish the following important property for $\mathcal{M}$.

Lemma 2.5 For any $u \in H^{1}\left(\mathbb{R}^{N}\right) \backslash\{0\}$, there is unique $t_{u}>0$ such that $u_{t_{u}} \in \mathcal{M}$.

Proof Let $u \in H^{1}\left(\mathbb{R}^{N}\right) \backslash\{0\}$ be fixed and define a function $\xi(t):=\mathcal{I}\left(u_{t}\right)$ on $(0, \infty)$. Clearly, by (1.11) and (2.7), we have

$$
\begin{aligned}
\xi^{\prime}(t)=0 \Leftrightarrow & \frac{N-2}{2} t^{N-2}\|\nabla u\|_{2}^{2}+\frac{t^{N}}{2} \int_{\mathbb{R}^{N}}[N V(t x)+\nabla V(t x) \cdot(t x)] u^{2} d x \\
& -\frac{N t^{N+\alpha}}{2} \int_{\mathbb{R}^{N}}\left(I_{\alpha} * u^{\frac{\alpha}{N}+1}\right) u^{\frac{\alpha}{N}+1} d x-\frac{N \lambda t^{N}}{p}\|u\|_{p}^{p}=0 \\
\Leftrightarrow & \mathcal{P}\left(u_{t}\right)=0 \Leftrightarrow u_{t} \in \mathcal{M} .
\end{aligned}
$$

It is not hard to verify, using (V1), (V2), (1.2), and (2.7), that $\lim _{t \rightarrow 0} \xi(t)=0, \xi(t)>0$ for $t>0$ small and $\xi(t)<0$ for $t$ large. Therefore $\max _{t \in(0, \infty)} \xi(t)$ is achieved at some $t_{u}>0$ so that $\xi^{\prime}\left(t_{u}\right)=0$ and $u_{t_{u}} \in \mathcal{M}$.

Not unnaturally, we claim that $t_{u}$ is unique for any $u \in H^{1}\left(\mathbb{R}^{N}\right) \backslash\{0\}$. As a matter of fact, for any given $u \in H^{1}\left(\mathbb{R}^{N}\right) \backslash\{0\}$, if there are two positive constants $t_{1} \neq t_{2}$ such that $u_{t_{1}}, u_{t_{2}} \in \mathcal{M}$, then $\mathcal{P}\left(u_{t_{1}}\right)=\mathcal{P}\left(u_{t_{2}}\right)=0$. Together with (2.3), (2.4), and (2.5), we have

$$
\begin{aligned}
\mathcal{I}\left(u_{t_{1}}\right) \geq & \mathcal{I}\left(u_{t_{2}}\right)+\frac{t_{1}^{N}-t_{2}^{N}}{N t_{1}^{N}} \mathcal{P}\left(u_{t_{1}}\right)+\frac{(1-\theta) g\left(t_{2} / t_{1}\right)}{2 N}\left\|\nabla u_{t_{1}}\right\|_{2}^{2} \\
& +\frac{\beta\left(t_{2} / t_{1}\right)}{2(N+\alpha)} \int_{\mathbb{R}^{N}}\left(I_{\alpha} * u_{t_{1}}^{\frac{\alpha}{N}+1}\right) u_{t_{1}}^{\frac{\alpha}{N}+1} d x \\
\geq & \mathcal{I}\left(u_{t_{2}}\right)+\frac{(1-\theta)\left[2 t_{1}^{N}-N t_{1}^{2} t_{2}^{N-2}+(N-2) t_{2}^{N}\right]}{2 N t_{1}^{2}}\|\nabla u\|_{2}^{2} .
\end{aligned}
$$


The same procedure may be easily adapted to obtain the following equation:

$$
\mathcal{I}\left(u_{t_{2}}\right) \geq \mathcal{I}\left(u_{t_{1}}\right)+\frac{(1-\theta)\left[2 t_{2}^{N}-N t_{2}^{2} t_{1}^{N-2}+(N-2) t_{1}^{N}\right]}{2 N t_{2}^{2}}\|\nabla u\|_{2}^{2}
$$

From (2.10) and (2.11), we have $u_{t_{1}}=u_{t_{2}}$, which shows that $t_{u}>0$ is unique for any $u \in H^{1}\left(\mathbb{R}^{N}\right) \backslash\{0\}$.

Lemma 2.6 ([19, Lemma 2.5]) Assume that (V1)-(V3) hold. Then there are two constants $\gamma_{1}, \gamma_{2}>0$ such that

$$
\gamma_{1}\|u\|^{2} \leq(N-2)\|\nabla u\|_{2}^{2}+\int_{\mathbb{R}^{N}}[N V(x)+\nabla V(x) \cdot x] u^{2} d x \leq \gamma_{2}\|u\|^{2} .
$$

Proof The proof of Lemma 2.6 is routine, and we omit it.

From Corollary 2.4 and Lemma 2.5 , we have $\mathcal{M} \neq \emptyset$. Next, we apply the method introduced in [26] to prove the following lemma, which is key to verifying the minimax characterization.

Lemma 2.7 Assume that (V1) and (V2) hold. Then

$$
\inf _{u \in \mathcal{M}} \mathcal{I}(u):=m=\inf _{u \in H^{1}(\mathbb{R}) \backslash\{0\}} \max _{t>0} \mathcal{I}\left(u_{t}\right)
$$

Lemma 2.8 Assume that (V1) and (V2) hold. Then

(i) there exists $\rho>0$ such that $\|u\|>\rho$.

(ii) $m=\inf _{u \in \mathcal{M}} \mathcal{I}(u)>0$.

Proof (i). Since $\mathcal{P}(u)=0$ for all $u \in \mathcal{M}$, by (2.1), (1.11), Lemma 2.6, and Sobolev embedding inequality, one has

$$
\begin{aligned}
\frac{\gamma_{1}}{2}\|u\|^{2} & \leq \frac{N-2}{2}\|\nabla u\|_{2}^{2}+\frac{1}{2} \int_{\mathbb{R}^{N}}[N V(x)+\nabla V(x) \cdot x] u^{2} d x \\
& =\frac{N}{2} \int_{\mathbb{R}^{N}}\left(I_{\alpha} *|u|^{\frac{\alpha}{N}+1}\right)|u|^{\frac{\alpha}{N}+1} d x+\frac{N \lambda}{p} \int_{\mathbb{R}^{N}}|u|^{p} d x \\
& \leq C_{1}\|u\|^{\frac{2(N+\alpha)}{N}}+C_{2}\|u\|^{p} .
\end{aligned}
$$

There are two cases to consider.

Case (1). When $\frac{2(N+\alpha)}{N} \geq p$, from (2.13), one has

$$
\frac{\gamma_{1}}{2}\|u\|^{2} \leq C_{1}\|u\|^{\frac{2(N+\alpha)}{N}}+C_{2}\|u\|^{\frac{2(N+\alpha)}{N}},
$$

which implies

$$
\|u\| \geq \rho_{0}:=\min \left\{1,\left[\frac{\gamma_{1}}{2\left(C_{1}+C_{2}\right)}\right]^{\frac{N}{2 \alpha}}\right\} .
$$


Case (2). When $p>\frac{2(N+\alpha)}{N}$ and $\lambda>0$, one has

$$
\frac{\gamma_{1}}{2}\|u\|^{2} \leq C_{1}\|u\|^{p}+C_{2}\|u\|^{p}
$$

which implies

$$
\|u\| \geq \rho_{1}:=\min \left\{1,\left[\frac{\gamma_{1}}{2\left(C_{1}+C_{2}\right)}\right]^{\frac{1}{p-2}}\right\}
$$

From (2.15) and (2.17), we know that (i) holds.

(ii). Let $\left\{u_{n}\right\} \subset \mathcal{M}$ be such that $\mathcal{I}\left(u_{n}\right) \rightarrow m$. There are two possible cases:

Case (i). $\inf _{n \in \mathbb{N}}\left\|\nabla u_{n}\right\|_{2}:=\sigma>0$. From (1.5) and (1.11), one has

$$
\begin{aligned}
\mathcal{I}\left(u_{n}\right)-\frac{1}{N} \mathcal{P}\left(u_{n}\right)= & \frac{1}{N}\left\|\nabla u_{n}\right\|_{2}^{2}-\frac{1}{2 N} \int_{\mathbb{R}^{N}} \nabla V(x) \cdot x u_{n}^{2} d x \\
& +\frac{\alpha}{2(N+\alpha)} \int_{\mathbb{R}^{N}}\left(I_{\alpha} *\left|u_{n}\right|^{\frac{\alpha}{N}+1}\right)\left|u_{n}\right|^{\frac{\alpha}{N}+1} d x, \quad \forall u \in H^{1}\left(\mathbb{R}^{N}\right) .
\end{aligned}
$$

From (V3), we have

$$
\int_{\mathbb{R}^{N}} \nabla V(x) \cdot x u^{2} d x \leq \frac{\theta(N-2)^{2}}{2} \int_{\mathbb{R}^{N}} \frac{u^{2}}{|x|^{2}} d x \leq 2 \theta\|\nabla u\|_{2}^{2}, \quad \forall u \in H^{1}\left(\mathbb{R}^{N}\right) .
$$

From (2.18) and (2.19), we obtain

$$
\begin{aligned}
m+o(1) & =\mathcal{I}\left(u_{n}\right)=\mathcal{I}\left(u_{n}\right)-\frac{1}{N} \mathcal{P}\left(u_{n}\right) \\
& \geq \frac{1}{N}\left\|\nabla u_{n}\right\|_{2}^{2}-\frac{\theta}{N}\left\|\nabla u_{n}\right\|_{2}^{2}+\frac{\alpha}{2(N+\alpha)} \int_{\mathbb{R}^{N}}\left(I_{\alpha} *\left|u_{n}\right|^{\frac{\alpha}{N}+1}\right)\left|u_{n}\right|^{\frac{\alpha}{N}+1} d x \\
& \geq \frac{1-\theta}{N}\left\|\nabla u_{n}\right\|_{2}^{2}=\frac{1-\theta}{N} \sigma^{2} .
\end{aligned}
$$

Case (ii). $\inf _{n \in \mathbb{N}}\left\|\nabla u_{n}\right\|_{2}=0$. In this case, by (2.15) and (2.17), passing to a subsequence, one has

$$
\left\|\nabla u_{n}\right\|_{2} \rightarrow 0, \quad\left\|u_{n}\right\|_{2} \geq \frac{1}{2} \max \left\{\rho_{0}, \rho_{1}\right\}
$$

By Lemma 2.1 and the Sobolev inequality, one has

$$
\int_{\mathbb{R}^{N}}\left(I_{\alpha} *\left|u_{n}\right|^{\frac{\alpha}{N}+1}\right)\left|u_{n}\right|^{\frac{\alpha}{N}+1} d x \leq C_{3}\left\|u_{n}\right\|_{2}^{\frac{2(N+\alpha)}{N}}
$$

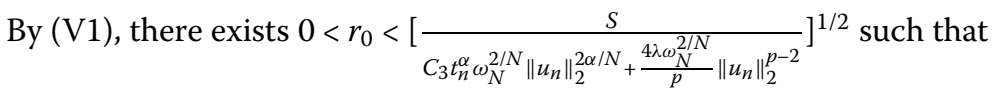

$$
V(x) \geq \frac{V_{\infty}}{2}>C_{3} t_{n}^{\alpha}\left\|u_{n}\right\|_{2}^{2 \alpha / N}+\frac{2 \lambda}{p}\left\|u_{n}\right\|_{2}^{p-2}
$$

for $|x| \geq r_{0}$. Then

$$
\int_{|t x| \geq r_{0}} V(t x) u_{n}^{2} d x \geq \frac{V_{\infty}}{2} \int_{|t x| \geq r_{0}} u_{n}^{2} d x, \quad \forall t>0, u_{n} \in H^{1}\left(\mathbb{R}^{N}\right) .
$$


By the Sobolev inequality and Hölder's inequality, we have

$$
\begin{aligned}
\int_{|t x|<r_{0}} u^{2} d x & \leq\left(\frac{\omega_{N} r_{0}^{N}}{t^{N}}\right)^{\left(2^{*}-2\right) / 2^{*}}\left(\int_{|t x|<r_{0}} u^{2^{*}} d x\right)^{2 / 2^{*}} \\
& \leq \omega_{N}^{2 / N} r_{0}^{2} t^{-2} S^{-1}\|\nabla u\|_{2}^{2}, \quad \forall t>0, u \in H^{1}\left(\mathbb{R}^{N}\right) .
\end{aligned}
$$

Let

$$
\sigma=\min \left\{V_{\infty}, S r_{0}^{-2} \omega_{N}^{-\frac{2}{N}}\right\}
$$

and

$$
t_{n}=\left(\frac{\sigma-\frac{4 \lambda}{p}\left\|u_{n}\right\|_{2}^{p-2}}{4 C_{3}}\right)^{\frac{1}{\alpha}}\left\|u_{n}\right\|_{2}^{-\frac{2}{N}}
$$

Since (2.21) implies that $\left\{t_{n}\right\}$ is bounded, then it follows from (2.7), (2.8), (2.21)-(2.27), Corollary 2.4, and the Sobolev embedding inequality that

$$
\begin{aligned}
m= & \mathcal{I}\left(u_{n}\right) \\
\geq & \mathcal{I}\left(\left(u_{n}\right)_{t_{n}}\right) \\
= & \frac{t_{n}^{N-2}}{2}\left\|\nabla u_{n}\right\|_{2}^{2}+\frac{t_{n}^{N}}{2} \int_{\mathbb{R}^{N}} V\left(t_{n} x\right) u_{n}^{2} d x-\frac{N t_{n}^{N+\alpha}}{2(N+\alpha)} \int_{\mathbb{R}^{N}}\left(I_{\alpha} *\left|u_{n}\right|^{\frac{\alpha}{N}+1}\right)\left|u_{n}\right|^{\frac{\alpha}{N}+1} d x \\
& -\frac{t_{n}^{N} \lambda}{p} \int_{\mathbb{R}^{N}}\left|u_{n}\right|^{p} d x \\
\geq & \frac{S t_{n}^{N}}{2 r_{0}^{2} \omega_{N}^{2 / N}} \int_{\left|t_{n} x\right|<r_{0}} u_{n}^{2} d x+\frac{t_{n}^{N} V_{\infty}}{4} \int_{\left|t_{n} x\right| \geq r_{0}} u_{n}^{2} d x-\frac{C_{3} t_{n}^{N+\alpha}}{2}\left\|u_{n}\right\|_{2}^{\frac{2(N+\alpha)}{N}}-\frac{\lambda t_{n}^{N}}{p}\left\|u_{n}\right\|_{2}^{p} \\
\geq & \frac{\sigma}{4}\left\|u_{n}\right\|_{2}^{2}-\frac{C_{3} t_{n}^{N+\alpha}}{2}\left\|u_{n}\right\|_{2}^{\frac{2(N+\alpha)}{N}}-\frac{\lambda t_{n}^{N}}{p}\left\|u_{n}\right\|_{2}^{p} \\
= & \frac{t_{n}^{N}}{4}\left\|u_{n}\right\|_{2}^{2}\left(\sigma-2 C_{3} t_{n}^{\alpha}\left\|u_{n}\right\|_{2}^{\frac{2 \alpha}{N}}-\frac{4 \lambda}{p}\left\|u_{n}\right\|_{2}^{p-2}\right)>0 .
\end{aligned}
$$

The two cases show that $m=\inf _{u \in \mathcal{M}} \mathcal{I}(u)>0$.

Inspired by Tang and Chen [25], we give an estimate on the energy level $m$, which is essential in ensuring compactness.

Lemma $2.9 m<m_{*}:=\frac{\alpha}{2(N+\alpha)}\left(V_{\infty} S\right)^{\frac{N}{\alpha}+1}$

Proof We set $U(x)=A_{0}\left(1+|x|^{2}\right)^{-\frac{N}{2}}$, where $A_{0}$ is defined by (1.4). By the calculation of integral, we get

$$
\|U\|_{p}^{p}=\int_{\mathbb{R}^{N}}|U|^{P} d x=\omega_{N} A_{0}^{p} \int_{0}^{+\infty} r^{N-1}\left(1+r^{2}\right)^{-p N / 2} d r=\frac{\omega_{N} A_{0}^{p} \Gamma\left(\frac{(p-1) N}{2}\right) \Gamma\left(\frac{N}{2}\right)}{\Gamma\left(\frac{p N}{2}\right)}
$$


and

$$
\|\nabla U\|_{2}^{2}=\int_{\mathbb{R}^{N}}|\nabla U|^{2} d x=\omega_{N}\left(N A_{0}\right)^{2} \int_{0}^{+\infty} r^{N+1}\left(1+r^{2}\right)^{-(N+2)} d r=\frac{\omega_{N}\left(N^{2} A_{0}\right)^{2}\left[\Gamma\left(\frac{N}{2}\right)\right]^{2}}{8(N+1) !} .
$$

Let $t_{*}=\left(V_{\infty} S\right)^{\frac{1}{\alpha}}$. For any $\varepsilon>0$, we define two functions $f(t)$ and $h_{\varepsilon}(t)$ as follows:

$$
f(t)=\frac{V_{\infty} S}{2} t^{N}-\frac{N}{2(N+\alpha)} t^{N+\alpha}
$$

and

$$
h_{\varepsilon}(t)=\frac{\varepsilon^{2}\|\nabla U\|_{2}^{2}}{2} t^{N-2}-\frac{\lambda \varepsilon^{\frac{N p}{2}-N}}{p}\|U\|_{p}^{p} t^{N} .
$$

It is easy to know that $f(t)<f\left(t_{*}\right)=\frac{\alpha}{2(N+\alpha)}\left(V_{\infty} S\right)^{\frac{N}{\alpha}+1}:=m_{*}$ for $t \in\left[0, t_{*}\right) \cup\left(t_{*}, \infty\right)$. We set $U_{\varepsilon}(x)=\varepsilon^{N / 2} U(\varepsilon x)$. Then it follows from the definition of $\mathcal{S}$ that

$$
\left\|U_{\varepsilon}\right\|_{2}^{2}=S \quad \text { and } \quad \int_{\mathbb{R}^{N}}\left(I_{\alpha} *|u|^{\frac{\alpha}{N}+1}\right)|u|^{\frac{\alpha}{N}+1} d x=1,
$$

and

$$
\left\|\nabla U_{\varepsilon}\right\|_{2}^{2}=\varepsilon^{2}\|\nabla U\|_{2}^{2} \quad \text { and } \quad\left\|U_{\varepsilon}\right\|_{p}^{p}=\varepsilon^{\frac{p N}{2}-N}\|U\|_{p}^{p} .
$$

From (2.7), (2.29), (2.30), (2.31), and (2.32), we obtain

$$
\begin{aligned}
\mathcal{I}\left(\left(U_{\varepsilon}\right)_{t}\right)= & \frac{t^{N-2}}{2}\left\|\nabla U_{\varepsilon}\right\|_{2}^{2}+\frac{t^{N}}{2} \int_{\mathbb{R}^{N}} V(t x) U_{\varepsilon}^{2} d x \\
& -\frac{N t^{N+\alpha}}{2(N+\alpha)} \int_{\mathbb{R}^{N}}\left(I_{\alpha} *\left|U_{\varepsilon}\right|^{\frac{\alpha}{N^{+}+1}}\right)\left|U_{\varepsilon}\right|^{\frac{\alpha}{N}+1} d x \\
& -\frac{t^{N} \lambda}{p} \int_{\mathbb{R}^{N}}\left|U_{\varepsilon}\right|^{p} d x \\
\leq & \frac{V_{\infty} t^{N}}{2}\left\|U_{\varepsilon}\right\|_{2}^{2}+\frac{t^{N-2}}{2}\left\|\nabla U_{\varepsilon}\right\|_{2}^{2}-\frac{N t^{N+\alpha}}{2(N+\alpha)} \int_{\mathbb{R}^{N}}\left(I_{\alpha} *\left|U_{\varepsilon}\right|^{\frac{\alpha}{N}+1}\right)\left|U_{\varepsilon}\right|^{\frac{\alpha}{N^{+1}}} d x \\
& -\frac{t^{N} \lambda}{p} \int_{\mathbb{R}^{N}}\left|U_{\varepsilon}\right|^{p} d x \\
= & \frac{V_{\infty} S}{2} t^{N}-\frac{N}{2(N+\alpha)} t^{N+\alpha}+\frac{\varepsilon^{2}\|\nabla U\|_{2}^{2}}{2} t^{N-2}-\frac{\lambda \varepsilon^{\frac{N p}{2}}-N}{p}\|U\|_{p}^{p} t^{N} \\
= & f(x)+h_{\varepsilon}(t) .
\end{aligned}
$$

There are three possible cases to distinguish.

Case 1. $2<p<2+\frac{4}{N}$ and $\lambda>0$. In this case, we choose $\varepsilon \in(0,1)$, then

$$
h_{\varepsilon}(t) \leq \frac{1}{2 p} \varepsilon^{2} t^{N-2}\left(p\|\nabla U\|_{2}^{2}-2 \lambda\|U\|_{p}^{p} t^{2}\right) .
$$


Let

$$
T_{0}=\max \left\{\frac{6 t_{*}}{5},\left(\frac{p\|\nabla U\|_{2}^{2}}{2 \lambda\|U\|_{p}^{p}}\right)^{\frac{1}{2}}\right\} .
$$

We can choose $\varepsilon \in(0,1)$ such that

$$
\lambda t_{*}^{2} \geq \frac{25 p\|\nabla U\|_{2}^{2}}{32\|U\|_{p}^{p}}
$$

and

$$
\frac{1}{2} \varepsilon^{2} T_{0}^{N-2}\|\nabla U\|_{2}^{2}<m_{*}-f\left(\frac{6 t_{*}}{5}\right), \quad \frac{1}{2} \varepsilon^{2} t_{*}^{N-2}\|\nabla U\|_{2}^{2}<m_{*}-f\left(\frac{4 t_{*}}{5}\right) .
$$

There are four possible subcases.

Subcase (i) $t \geq T_{0}$. Then it follows from (2.29), (2.33), (2.34), and (2.35) that

$$
\begin{aligned}
\max _{t \geq T_{0}} \mathcal{I}\left(\left(U_{\varepsilon}\right)_{t}\right) & \leq \max _{t \geq T_{0}}\left[f(t)+h_{\varepsilon}(t)\right] \\
& \leq f\left(\frac{6 t_{*}}{5}\right) \leq f\left(t_{*}\right)=m_{*} .
\end{aligned}
$$

Subcase (ii) $\frac{6 t_{*}}{5} \leq t \leq T_{0}$. Then it follows from (2.29), (2.33), (2.34), and (2.37) that

$$
\begin{aligned}
\max _{\frac{6 *_{*}}{5} \leq t \leq T_{0}} \mathcal{I}\left(\left(U_{\varepsilon}\right)_{t}\right) & \leq \max _{\frac{6 t_{*}}{5} \leq t \leq T_{0}}\left[f(t)+h_{\varepsilon}(t)\right] \\
& \leq f\left(\frac{6 t_{*}}{5}\right)+\frac{1}{2} \varepsilon_{2} T_{0}^{N-2}\|\nabla U\|_{2}^{2}<m_{*} .
\end{aligned}
$$

Subcase (iii) $\frac{4 t_{*}}{5} \leq t \leq \frac{6 t_{*}}{5}$. Then it follows from (2.29), (2.33), and (2.35) that

$$
\begin{aligned}
\max _{\frac{4 t_{*}}{5} \leq t \leq \frac{6 t_{*}}{5}} \mathcal{I}\left(\left(U_{\varepsilon}\right)_{t}\right) & \leq \max _{\frac{4 t_{*}}{5} \leq t \leq \frac{6 t_{*}}{5}}\left[f(t)+h_{\varepsilon}(t)\right] \\
& \leq f\left(t_{*}\right)+h_{\varepsilon}\left(\frac{4 t_{*}}{5}\right) \leq m_{*} .
\end{aligned}
$$

Subcase (iv) $0 \leq t \leq \frac{4 t_{*}}{5}$. Then it follows from (2.29), (2.33), (2.34), and (2.37) that

$$
\begin{aligned}
\max _{0 \leq t \leq \frac{4 t_{*}}{5}} \mathcal{I}\left(\left(U_{\varepsilon}\right)_{t}\right) & \leq \max _{0 \leq t \leq \frac{4 t_{*}}{5}}\left[f(t)+h_{\varepsilon}(t)\right] \\
& \leq f\left(\frac{4 t_{*}}{5}\right)+\frac{1}{2} \varepsilon_{2} t_{*}^{N-2}\|\nabla U\|_{2}^{2}<m_{*} .
\end{aligned}
$$

Case 2. $p=2+\frac{4}{N}$ and $\lambda>\frac{(N+2) N^{2}}{2(N+1) A_{0}^{\frac{4}{N}}\left(S V_{\infty}\right)^{\frac{2}{\alpha}}}$. In this case we choose $\varepsilon \in(0,1)$, then

$$
h_{\varepsilon}(t)=\frac{1}{2} \varepsilon^{2} t^{N-2}\left(\|\nabla U\|_{2}^{2}-\frac{N}{N+2} \lambda\|U\|_{2+4 / N}^{2+4 / N} t^{2}\right) .
$$


Let

$$
T_{1}=\max \left\{t_{*}+\epsilon,\left(\frac{(N+2)\|\nabla U\|_{2}^{2}}{N \lambda\|U\|_{2+4 / N}^{2+4 / N}}\right)^{\frac{1}{2}}\right\} .
$$

By assumption (ii) in Theorem 1.1, we can choose $\epsilon>0$ such that

$$
\lambda\left(t_{*}-\epsilon\right)^{2} \geq \frac{(N+2)\|\nabla U\|_{2}^{2}}{N\|U\|_{2+4 / N}^{2+4 / N}} .
$$

We choose $\varepsilon>0$ such that

$$
\frac{1}{2} \varepsilon^{2} T_{1}^{N-2}\|\nabla U\|_{2}^{2}<m_{*}-f\left(t_{*}+\epsilon\right), \quad \frac{1}{2} \varepsilon^{2} t_{*}^{N-2}\|\nabla U\|_{2}^{2}<m_{*}-f\left(t_{*}-\epsilon\right) .
$$

There are also four possible subcases.

Subcase (i) $t \geq T_{1}$. Then it follows from (2.29), (2.33), (2.42), and (2.43) that

$$
\begin{aligned}
\max _{t \geq T_{1}} \mathcal{I}\left(\left(U_{\varepsilon}\right)_{t}\right) & \leq \max _{t \geq T_{1}}\left[f(t)+h_{\varepsilon}(t)\right] \\
& \leq f\left(t_{*}+\epsilon\right) \leq f\left(t_{*}\right)=m_{*} .
\end{aligned}
$$

Subcase (ii) $t_{*}+\epsilon \leq t \leq T_{1}$. Then it follows from (2.29), (2.33), (2.42), and (2.45) that

$$
\begin{aligned}
\max _{t_{*}+\epsilon \leq t \leq T_{1}} \mathcal{I}\left(\left(U_{\varepsilon}\right)_{t}\right) & \leq \max _{t_{*}+\epsilon \leq t \leq T_{1}}\left[f(t)+h_{\varepsilon}(t)\right] \\
& \leq f\left(t_{*}+\epsilon\right)+\frac{1}{2} \varepsilon_{2} T_{1}^{N-2}\|\nabla U\|_{2}^{2}<m_{*} .
\end{aligned}
$$

Subcase (iii) $t_{*}-\epsilon \leq t \leq t_{*}+\epsilon$. Then it follows from (2.29), (2.33), (2.42), and (2.44) that

$$
\begin{aligned}
\max _{t_{*}-\epsilon \leq t \leq t_{*}+\epsilon} \mathcal{I}\left(\left(U_{\varepsilon}\right)_{t}\right) & \leq \max _{t_{*}-\epsilon \leq t \leq t_{*}+\epsilon}\left[f(t)+h_{\varepsilon}(t)\right] \\
& \leq f\left(t_{*}\right)+h_{\varepsilon}\left(t_{*}-\epsilon\right) \leq m_{*} .
\end{aligned}
$$

Subcase (iv) $0 \leq t \leq t_{*}-\epsilon$. Then it follows from (2.29), (2.33), (2.42), and (2.45) that

$$
\begin{aligned}
\max _{0 \leq t \leq t_{*}-\epsilon} \mathcal{I}\left(\left(U_{\varepsilon}\right)_{t}\right) & \leq \max _{0 \leq t \leq t_{*}-\epsilon}\left[f(t)+h_{\varepsilon}(t)\right] \\
& \leq f\left(t_{*}-\epsilon\right)+\frac{1}{2} \varepsilon^{2} t_{*}^{N-2}\|\nabla U\|_{2}^{2}<m_{*} .
\end{aligned}
$$

Case 3. $2+\frac{4}{N}<p<2^{*}$ and $\lambda>\frac{25 p N^{4} \Gamma\left(\frac{N}{2}\right) \Gamma\left(\frac{p N}{2}\right)}{256(N+1) ! A_{0}^{p-2} \varepsilon^{2^{*}-2}\left(V_{\infty} S\right)^{\frac{2}{\alpha}} \Gamma\left(\frac{(p-1) N}{2}\right)}$. In this case, we also choose $\varepsilon \in(0,1]$, then

$$
\begin{gathered}
h_{\varepsilon}(t) \leq \frac{\varepsilon^{2}\|\nabla U\|_{2}^{2}}{2} t^{N-2}-\frac{\lambda \varepsilon^{2^{*}}}{p}\|U\|_{p}^{p} t^{N}, \\
T_{2}=\max \left\{\frac{6 t_{*}}{5},\left(\frac{p\|\nabla U\|_{2}^{2}}{2 \lambda \varepsilon^{2^{*}-2}\|U\|_{p}^{p}}\right)^{1 / 2}\right\},
\end{gathered}
$$


and

$$
\lambda>\frac{p\|\nabla U\|_{2}^{2}}{2 \varepsilon^{\frac{(p-2) N}{2}-2} t_{0}^{2}} .
$$

Now we can choose $\varepsilon \in(0,1]$ and $\lambda>0$ such that

$$
\lambda t_{*}^{2} \geq \frac{25 p\|\nabla U\|_{2}^{2}}{32 \varepsilon^{2^{*}-2}\|U\|_{p}^{p}}
$$

and

$$
\frac{1}{2} \varepsilon^{2} T_{2}^{N-2}\|\nabla U\|_{2}^{2}<m_{*}-f\left(\frac{6 t_{*}}{5}\right), \quad \frac{1}{2} \varepsilon^{2} t_{*}^{N-2}\|\nabla U\|_{2}^{2}<m_{*}-f\left(\frac{4 t_{*}}{5}\right) .
$$

There are four possible subcases.

Subcase (i) $t \geq T_{2}$. Then it follows from (2.29), (2.33), (2.51), and (2.50) that

$$
\begin{aligned}
\max _{t \geq T_{2}} \mathcal{I}\left(\left(U_{\varepsilon}\right)_{t}\right) & \leq \max _{t \geq T_{2}}\left[f(t)+h_{\varepsilon}(t)\right] \\
& \leq f\left(\frac{6 t_{*}}{5}\right) \leq f\left(t_{*}\right)=m_{*} .
\end{aligned}
$$

Subcase (ii) $\frac{6 t_{*}}{5} \leq t \leq T_{2}$. Then it follows from (2.29), (2.33), (2.52), (2.50), and (2.54) that

$$
\begin{aligned}
\max _{\frac{\sigma_{*}}{5} \leq t \leq T_{2}} \mathcal{I}\left(\left(U_{\varepsilon}\right)_{t}\right) & \leq \max _{\frac{6 \sigma_{*}}{5} \leq t \leq T_{2}}\left[f(t)+h_{\varepsilon}(t)\right] \\
& \leq f\left(\frac{6 t_{*}}{5}\right)+\frac{1}{2} \varepsilon^{2} T_{2}^{N-2}\|\nabla U\|_{2}^{2}<m_{*} .
\end{aligned}
$$

Subcase (iii) $\frac{4 t_{*}}{5} \leq t \leq \frac{6 t_{*}}{5}$. Then it follows from (2.29), (2.50), and (2.53) that

$$
\begin{aligned}
\max _{\frac{4 t_{*}}{5} \leq t \leq \frac{6 t_{*}}{5}} \mathcal{I}\left(\left(U_{\varepsilon}\right)_{t}\right) & \leq \max _{\frac{4 t_{*}}{5} \leq t \leq \frac{6 t_{*}}{5}}\left[f(t)+h_{\varepsilon}(t)\right] \\
& \leq f\left(t_{*}\right)+h_{\varepsilon}\left(\frac{4 t_{*}}{5}\right) \leq m_{*} .
\end{aligned}
$$

Subcase (iv) $0 \leq t \leq \frac{4 t_{*}}{5}$. Then it follows from (2.29), (2.33), (2.50), and (2.54) that

$$
\begin{aligned}
\max _{0 \leq t \leq \frac{4 t_{*}}{5}} \mathcal{I}\left(\left(U_{\varepsilon}\right)_{t}\right) & \leq \max _{0 \leq t \leq \frac{4 t_{*}}{5}}\left[f(t)+h_{\varepsilon}(t)\right] \\
& \leq f\left(\frac{4 t_{*}}{5}\right)+\frac{1}{2} \varepsilon^{2} t_{*}^{N-2}\|\nabla U\|_{2}^{2}<m_{*} .
\end{aligned}
$$

The above three cases show that

$$
m \leq \max _{t>0} \mathcal{I}\left(\left(U_{\varepsilon}\right)_{t}\right)<m_{*} .
$$

Lemma 2.10 Assume that (V1)-(V3) hold. Then $m$ is achieved. 
Proof In view of Lemmas 2.5 and 2.8, we have $\mathcal{M} \neq \emptyset$ and $m>0$. Let $\left\{u_{n}\right\} \subset \mathcal{M}$ be such that $\mathcal{I}\left(u_{n}\right) \rightarrow m$. Since $\mathcal{P}\left(u_{n}\right)=0$, then it follows from (2.5), (2.18), and (2.20) that

$$
\begin{aligned}
m+o(1) & =\mathcal{I}\left(u_{n}\right) \geq \mathcal{I}\left(u_{n}\right)-\frac{1}{N} \mathcal{P}\left(u_{n}\right) \\
& \geq \frac{1-\theta}{N}\left\|\nabla u_{n}\right\|_{2}^{2} .
\end{aligned}
$$

This is to show that $\left\{\left\|\nabla u_{n}\right\|_{2}\right\}$ is bounded. Next, we prove that $\left\{\left\|u_{n}\right\|_{2}\right\}$ is also bounded. Arguing indirectly, assume that $\left\|u_{n}\right\|_{2} \rightarrow \infty$, without loss of generality, we can assume that $\left\|u_{n}\right\|_{2} \geq 1$. From (2.28), we have

$$
\begin{aligned}
m= & \mathcal{I}\left(u_{n}\right) \geq \mathcal{I}\left(\left(u_{n} \bar{t}_{n}\right)\right. \\
= & \frac{\bar{t}_{n}^{N-2}}{2}\left\|\nabla u_{n}\right\|_{2}^{2}+\frac{\bar{t}_{n}^{N}}{2} \int_{\mathbb{R}^{N}} V\left(\bar{t}_{n} x\right) u_{n}^{2} d x-\frac{\bar{t}_{n}^{N} \lambda}{p} \int_{\mathbb{R}^{N}}\left|u_{n}\right|^{p} d x \\
& -\frac{N \bar{t}_{n}^{N+\alpha}}{2(N+\alpha)} \int_{\mathbb{R}^{N}}\left(\mathcal{I}_{\alpha} *\left|u_{n}\right|^{\frac{\alpha}{N}+1}\right)\left|u_{n}\right|^{\frac{\alpha}{N}+1} d x \\
\geq & \frac{S \bar{t}_{n}^{N}}{2 r_{0} \omega_{N}^{2 / N}} \int_{\left|\bar{t}_{n} x\right|<r_{0}} u_{n}^{2} d x+\frac{V_{\infty} \bar{t}_{n}^{N}}{4} \int_{\left|\bar{t}_{n} x\right| \geq r_{0}} u_{n}^{2} d x-\frac{C_{3} \bar{t}_{n}^{N+\alpha}}{2}\left\|u_{n}\right\|_{2}^{\frac{2(N+\alpha)}{N}}-\frac{\lambda \bar{t}_{n}^{N}}{p}\left\|u_{n}\right\|_{2}^{p} \\
\geq & \frac{\sigma \bar{t}_{n}^{N}}{4}\left\|u_{n}\right\|_{2}^{2}-\frac{C_{3} \bar{t}^{N+\alpha}}{2}\left\|u_{n}\right\|_{2}^{\frac{2(N+\alpha)}{N}}-\frac{\lambda \bar{t}_{n}^{N}}{p}\left\|u_{n}\right\|_{2}^{p} \\
= & \frac{\bar{t}_{n}^{N}}{4}\left\|u_{n}\right\|_{2}^{2}\left(\sigma-2 C_{3} \bar{t}_{n}^{\alpha}\left\|u_{n}\right\|_{2}^{\frac{2 \alpha}{N}}-\frac{4 \lambda}{p}\left\|u_{n}\right\|_{2}^{p-2}\right), \quad \forall \bar{t}_{n} \geq 0 .
\end{aligned}
$$

If $\frac{2 \alpha}{N} \geq p-2$, we choose

$$
\lambda=\frac{p C_{3} \bar{t}_{n}^{\alpha}}{4}>0 \quad \text { and } \quad C_{3}=\frac{\sigma}{4}\left(\frac{\sigma}{24 m}\right)^{\frac{\alpha}{N}} .
$$

Let

$$
\bar{t}_{n}=\left(\frac{24 m}{\sigma}\right)^{\frac{1}{N}}\left\|u_{n}\right\|_{2}^{-\frac{2}{N}} .
$$

From (2.61), (2.62), and (2.63), we have

$$
\begin{aligned}
m & \geq \frac{\bar{t}_{n}^{N}}{4}\left\|u_{n}\right\|_{2}^{2}\left(\sigma-2 C_{3} \bar{t}_{n}^{\alpha}\left\|u_{n}\right\|_{2}^{\frac{2 \alpha}{N}}-\frac{4 \lambda}{p}\left\|u_{n}\right\|_{2}^{p-2}\right) \\
& \geq \frac{\bar{t}_{n}^{N}}{4}\left\|u_{n}\right\|_{2}^{2}\left(\sigma-2 C_{3} \bar{t}_{n}^{\alpha}\left\|u_{n}\right\|_{2}^{\frac{2 \alpha}{N}}-\frac{4 \lambda}{p}\left\|u_{n}\right\|_{2}^{\frac{2 \alpha}{N}}\right) \\
& =\frac{3}{2} m .
\end{aligned}
$$

This is a contradiction.

When $\frac{2 \alpha}{N}<p-2$, we choose

$$
\lambda=\frac{p C_{3} \bar{t}_{n}^{\alpha}}{4}>0 \quad \text { and } \quad C_{3}=\frac{\sigma}{4}\left(\frac{\sigma}{24 m\left\|u_{n}\right\|_{2}^{[N(p-2)-2 \alpha] / \alpha}}\right)^{\alpha / N} .
$$


Let

$$
\bar{t}_{n}=\left(\frac{24 m\left\|u_{n}\right\|_{2}^{[N(p-2)-2 \alpha] / \alpha}}{\sigma}\right)^{1 / N}\left\|u_{n}\right\|^{-(p-2) / \alpha} .
$$

Then from (2.61), (2.65), and (2.66), we get

$$
\begin{aligned}
m & \geq \frac{\bar{t}_{n}^{N}}{4}\left\|u_{n}\right\|_{2}^{2}\left(\sigma-2 C_{3} \bar{t}_{n}^{\alpha}\left\|u_{n}\right\|_{2}^{2 \alpha}-\frac{4 \lambda}{p}\left\|u_{n}\right\|_{2}^{p-2}\right) \\
& \geq \frac{\bar{t}_{n}^{N}}{4}\left\|u_{n}\right\|_{2}^{2}\left(\sigma-2 C_{3} \bar{t}_{n}^{\alpha}\left\|u_{n}\right\|_{2}^{p-2}-\frac{4 \lambda}{p}\left\|u_{n}\right\|_{2}^{p-2}\right) \\
& =\frac{3}{2} m,
\end{aligned}
$$

a contradiction. Hence, $\left\{\left\|u_{n}\right\|_{2}\right\}$ is also bounded. Therefore, $\left\{u_{n}\right\}$ is bounded in $H^{1}\left(\mathbb{R}^{N}\right)$. Passing to a subsequence, we have $u_{n} \rightarrow \bar{u}$ in $H^{1}\left(\mathbb{R}^{N}\right)$. Then $u_{n} \rightarrow \bar{u}$ in $L_{\mathrm{loc}}^{s}\left(\mathbb{R}^{N}\right)$ for $2 \leq$ $s \leq 2^{*}$ and $u_{n} \rightarrow \bar{u}$ a.e. in $\mathbb{R}^{N}$. We obtain two possible cases.

Case (i) $\bar{u}=0$, i.e., $u_{n} \rightarrow 0$ in $H^{1}\left(\mathbb{R}^{N}\right)$. Then $u_{n} \rightarrow 0$ in $L_{\text {loc }}^{s}\left(\mathbb{R}^{N}\right)$ for $2 \leq s \leq 2^{*}$ and $u_{n} \rightarrow 0$ a.e. in $\mathbb{R}^{N}$. Let $t=0$ in (2.4), one has

$$
N V(x)+\nabla V(x) \cdot x \leq N V_{\infty}+\frac{(N-2)^{2} \theta}{2|x|^{2}} .
$$

Let $t \rightarrow \infty$ in (2.4), one has

$$
-\frac{(N-2)^{3} \theta}{4|x|^{2}}+N V_{\infty} \leq N V(x)+\nabla V(x) \cdot x .
$$

By (V2), (2.68), and (2.69), it is easy to show that

$$
\lim _{n \rightarrow \infty} \int_{\mathbb{R}^{N}}\left[V_{\infty}-V(x)\right] u_{n}^{2} d x=\lim _{n \rightarrow \infty} \int_{\mathbb{R}^{N}} \nabla V(x) \cdot x u_{n}^{2} d x=0 .
$$

From (1.5), (1.8), (1.11), and (2.70), one can get

$$
\mathcal{I}\left(u_{n}\right) \rightarrow m, \quad \mathcal{P}^{\infty}\left(u_{n}\right) \rightarrow 0 .
$$

From Lemma 2.8(i), (1.12), and (2.71), one has

$$
\begin{aligned}
\min \left\{N-2, N V_{\infty}\right\} \rho^{2} & \leq \min \left\{N-2, N V_{\infty}\right\}\left\|u_{n}\right\|^{2} \\
& \leq(N-2)\left\|\nabla u_{n}\right\|_{2}^{2}+N V_{\infty}\left\|u_{n}\right\|_{2}^{2} \\
& =N \int_{\mathbb{R}^{N}}\left(I_{\alpha} *\left|u_{n}\right|^{\frac{\alpha}{N}+1}\right)\left|u_{n}\right|^{\frac{\alpha}{N}+1} d x+\frac{2 N \lambda}{p}\left\|u_{n}\right\|_{p}^{p} .
\end{aligned}
$$

Using (2.72) and the Lions concentration compactness principle [14, Lemma 1.21], we can prove that there exist $\sigma>0$ and a sequence $\left\{y_{n}\right\} \subset \mathbb{R}^{N}$ such that $\int_{B_{1}\left(y_{n}\right)}\left|u_{n}\right|^{2} d x>\sigma$. Let $\hat{u}_{n}(x)=u_{n}\left(x+y_{n}\right)$. Then we have $\left\|\hat{u}_{n}\right\|=\left\|u_{n}\right\|$ and

$$
\mathcal{P}^{\infty}\left(\hat{u}_{n}\right)=o(1), \quad \mathcal{I}\left(\hat{u}_{n}\right) \rightarrow m, \quad \int_{B_{1}(0)}\left|\hat{u}_{n}\right|^{2} d x>\sigma .
$$


Therefore, there exists $\hat{u} \in H^{1}\left(\mathbb{R}^{N}\right) \backslash\{0\}$ such that, passing to a subsequence,

$$
\begin{cases}\hat{u}_{n} \rightarrow \hat{u} & \text { in } H^{1}\left(\mathbb{R}^{N}\right) ; \\ \hat{u}_{n} \rightarrow \hat{u} & \text { in } L_{\text {loc }}^{s}\left(\mathbb{R}^{N}\right), \forall s \in\left[1,2^{*}\right) ; \\ \hat{u}_{n} \rightarrow \hat{u} & \text { a.e. on } \mathbb{R}^{N} .\end{cases}
$$

Let $w_{n}=\hat{u}_{n}-\hat{u}$. Then (2.74) and the Brezis-Lieb type lemma (see [11, Lemmas 2.4]), [30, Lemmas 2.10] lead to

$$
\mathcal{I}^{\infty}\left(\hat{u}_{n}\right)=\mathcal{I}^{\infty}(\hat{u})+\mathcal{I}^{\infty}\left(w_{n}\right)+o(1)
$$

and

$$
\mathcal{P}^{\infty}\left(\hat{u}_{n}\right)=\mathcal{P}^{\infty}(\hat{u})+\mathcal{P}^{\infty}\left(w_{n}\right)+o(1)
$$

From (1.12), (1.8), and Lemma 2.3, one has

$$
\mathcal{I}^{\infty}(u) \geq \mathcal{I}^{\infty}\left(u_{t}\right)
$$

Moreover,

$$
\begin{aligned}
& \frac{1}{N}\left\|\nabla w_{n}\right\|_{2}^{2}=m-\frac{1}{N}\|\nabla \hat{u}\|_{2}^{2}+\frac{\alpha}{2(N+\alpha)} \int_{\mathbb{R}^{N}}\left(I_{\alpha} *|\hat{u}|^{\frac{\alpha}{N}+1}\right)|\hat{u}|^{\frac{\alpha}{N}+1} d x+o(1), \\
& \mathcal{P}^{\infty}\left(w_{n}\right)=-\mathcal{P}^{\infty}(\hat{u})+o(1) .
\end{aligned}
$$

If there exists a subsequence $\left\{w_{n_{i}}\right\}$ of $\left\{w_{n}\right\}$ such that $w_{n_{i}}=0$, then going to this subsequence, we have

$$
\mathcal{I}^{\infty}(\hat{u})=m, \quad \mathcal{P}^{\infty}(\hat{u})=0 .
$$

Next we assume that $w_{n} \neq 0$. We claim that $\mathcal{P}^{\infty}(\hat{u}) \leq 0$. Otherwise, $\mathcal{P}^{\infty}(\hat{u})>0$ for large $n$. In view of Corollary 2.4 and Lemma 2.5 , there exists $t_{n}>0$ such that $\left(w_{n}\right)_{t_{n}} \in \mathcal{M}^{\infty}$. From (1.5), (1.12), (2.77), (2.78), and (2.80), we obtain

$$
\begin{aligned}
m-\frac{1}{N}\left\|\nabla \omega_{n}\right\|_{2}^{2}+o(1) & =\frac{1}{N}\|\nabla \hat{u}\|_{2}^{2}+\frac{\alpha}{2(N+\alpha)} \int_{\mathbb{R}^{N}}\left(I_{\alpha} *|\hat{u}|^{\frac{\alpha}{N}+1}\right)|\hat{u}|^{\frac{\alpha}{N}+1} d x \\
& =\mathcal{I}^{\infty}\left(w_{n}\right)-\frac{1}{N} \mathcal{P}^{\infty}\left(w_{n}\right) \\
& \geq \mathcal{I}^{\infty}\left(\left(w_{n}\right)_{t_{n}}\right)-\frac{t_{n}^{N}}{N} \mathcal{P}^{\infty}\left(w_{n}\right) \\
& \geq m^{\infty}-\frac{t_{n}^{N}}{N} \mathcal{P}^{\infty}\left(w_{n}\right) \geq m^{\infty},
\end{aligned}
$$

which implies $\mathcal{P}^{\infty}(\hat{u}) \leq 0$ due to $\|\nabla \hat{u}\|_{2}>0$. Since $\hat{u} \neq 0$ and $\mathcal{P}^{\infty}(\hat{u}) \leq 0$, in view of Lemma 2.5 , there exists $\hat{t}>0$ such that $\hat{u}_{\hat{t}} \in \mathcal{M}^{\infty}$. From (1.8), (1.12), (2.77), (2.78), (2.80) and the weak semicontinuity of norm, one has

$$
m=\lim _{n \rightarrow \infty}\left[\mathcal{I}^{\infty}\left(\hat{u}_{n}\right)-\frac{1}{N} \mathcal{P}^{\infty}\left(\hat{u}_{n}\right)\right]
$$




$$
\begin{aligned}
&=\frac{1}{N} \lim _{n \rightarrow \infty}\left\|\nabla \hat{u}_{n}\right\|_{2}^{2}+\frac{\alpha}{2(N+\alpha)} \lim _{n \rightarrow \infty} \int_{\mathbb{R}^{N}}\left(I_{\alpha} *\left|\hat{u}_{n}\right|^{\frac{\alpha}{N}+1}\right)\left|\hat{u}_{n}\right|^{\frac{\alpha}{N}+1} d x \\
& \geq \mathcal{I}^{\infty}(\hat{u})-\frac{1}{N} \mathcal{P}^{\infty}(\hat{u}) \geq \mathcal{I}^{\infty}\left(\hat{u}_{\hat{t}}\right)-\frac{\hat{t}^{N}}{N} \mathcal{P}^{\infty}(\hat{u}) \\
& \geq m^{\infty}-\frac{\hat{t}^{N}}{N} \mathcal{P}^{\infty}(\hat{u}) \geq m,
\end{aligned}
$$

which implies that (2.80) also holds. In view of Lemma 2.5, there exists $\hat{t}>0$ such that $\hat{u}_{\hat{t}} \in \mathcal{M}$; moreover, it follows from (V2), (1.5), (1.8), (2.81), and (2.82) that

$$
m \leq \mathcal{I}\left(\hat{u}_{\hat{t}}\right) \leq \mathcal{I}^{\infty}\left(\hat{u}_{\hat{t}}\right) \leq \mathcal{I}^{\infty}(\hat{u})=m
$$

This shows that $m$ is achieved at $\hat{u}_{\hat{t}} \in \mathcal{M}$.

Case (ii). $\bar{u} \neq 0$. Let $v_{n}=u_{n}-\bar{u}$. If $u_{n} \rightarrow \bar{u}$, similar to [17] and [31], we have the following two equalities:

$$
\mathcal{I}\left(u_{n}\right)=\mathcal{I}(\bar{u})+\mathcal{I}\left(v_{n}\right)+o(1)
$$

and

$$
\mathcal{P}\left(u_{n}\right)=\mathcal{P}(\bar{u})+\mathcal{P}\left(v_{n}\right)+o(1) .
$$

Set

$$
\begin{aligned}
\Psi(u)= & \frac{1}{N}\|\nabla u\|_{2}^{2}-\frac{1}{2 N} \int_{\mathbb{R}^{N}}(\nabla V(x) \cdot x) u^{2} d x \\
& +\frac{\alpha}{2(N+\alpha)} \int_{\mathbb{R}^{N}}\left(I_{\alpha} * u^{\frac{\alpha}{N}+1}\right) u^{\frac{\alpha}{N}+1} d x .
\end{aligned}
$$

Then it follows from (1.2), (2.4) with $t=0,(2.6)$ and (2.86) that

$$
\Psi(u) \geq \frac{1-\theta}{N}\|\nabla u\|_{2}^{2}, \quad \forall u \in H^{1}\left(\mathbb{R}^{N}\right) .
$$

Since $\mathcal{I}\left(u_{n}\right) \rightarrow m$ and $\mathcal{P}\left(u_{n}\right)=0$, then it follows from (1.5), (1.11), (2.85), (2.86), and (2.87) that

$$
\Psi\left(v_{n}\right)=m-\Psi(\bar{u})+o(1)
$$

and

$$
\mathcal{P}\left(v_{n}\right)=-\mathcal{P}(\bar{u})+o(1) .
$$

If there exists a subsequence $\left\{v_{n_{i}}\right\}$ of $\left\{v_{n}\right\}$ such that $v_{n_{i}}=0$, then going to this subsequence, we have

$$
\mathcal{I}(\bar{u})=m, \quad \mathcal{P}(\bar{u})=0,
$$


which implies that the conclusion of Lemma 2.10 holds. Next, we assume that $v_{n} \neq 0$. We claim that $\mathcal{P}(\bar{u}) \leq 0$. Otherwise, $\mathcal{P}(\bar{u})>0$, then (2.88) implies $\mathcal{P}\left(v_{n}\right)<0$ for large $n$. In view of (2.8), there exists $t_{n}>0$ such that $\left(v_{n}\right)_{t_{n}} \in \mathcal{M}$ for large $n$. From (1.5), (1.11), (2.5), (2.88), and (2.89), we obtain

$$
\begin{aligned}
m-\Psi(\bar{u})+o(1) & =\Psi\left(v_{n}\right) \\
& =\mathcal{I}\left(v_{n}\right)-\frac{1}{N} \mathcal{P}\left(v_{n}\right) \\
& \geq \mathcal{I}\left(\left(v_{n}\right)_{t_{n}}\right)-\frac{t_{n}^{N}}{N} \mathcal{P}\left(v_{n}\right) \\
& \geq m-\frac{t_{n}^{N}}{N} \mathcal{P}\left(v_{n}\right) \geq m,
\end{aligned}
$$

which implies $\mathcal{P}(\bar{u}) \leq 0$ due to $\Psi(\bar{u})>0$. Since $\bar{u} \neq 0$ and $\mathcal{P}(\bar{u}) \leq 0$, in view of (2.8) and (2.77), there exists $\bar{t}>0$ such that $\bar{u}_{\bar{t}} \in \mathcal{M}$. From (1.5), (1.11), (2.5), (2.87), (2.88) and the weak semicontinuity of norm, we obtain

$$
\begin{aligned}
m & =\lim _{n \rightarrow \infty}\left[\mathcal{I}\left(u_{n}\right)-\frac{1}{N} \mathcal{P}\left(u_{n}\right)\right]=\lim _{n \rightarrow \infty} \Psi\left(u_{n}\right) \geq \Psi(\bar{u}) \\
& \geq \mathcal{I}(\bar{u})-\frac{1}{N} \mathcal{P}(\bar{u}) \geq \mathcal{I}\left(\bar{u}_{\bar{t}}\right)-\frac{\bar{t}^{N}}{N} \mathcal{P}(\bar{u}) \\
& \geq m-\frac{\bar{t}^{N}}{N} \mathcal{P}^{\infty}(\bar{u}) \geq m,
\end{aligned}
$$

which implies that (2.90) also holds.

Lemma 2.11 Assume that $(V 1)-(V 3)$ hold. If $\bar{u} \in \mathcal{M}$ and $\mathcal{I}(\bar{u})=m$, then $\bar{u}$ is a critical point of $\mathcal{I}$.

Proof Similar to the proof of [32, Lemma 2.12], we can clearly conclude the desired conclusion by using

$$
\begin{aligned}
\mathcal{I}\left(\bar{u}_{t}\right) & \leq \mathcal{I}(\bar{u})-\frac{(1-\theta) g(t)}{2 N}\|\nabla \bar{u}\|_{2}^{2}-\frac{\beta(t)}{2(N+\alpha)} \int_{\mathbb{R}^{N}}\left(I_{\alpha} *|\bar{u}|^{\frac{\alpha}{N}+1}\right)|\bar{u}|^{\frac{\alpha}{N}+1} d x \\
& \leq \mathcal{I}(\bar{u})-\frac{(1-\theta) g(t)}{2 N}\|\nabla \bar{u}\|_{2}^{2}<m, \quad \forall t>0,
\end{aligned}
$$

and

$$
\varepsilon_{1}:=\min \left\{\frac{(1-\theta) g\left(1-\epsilon_{1}\right)}{5(N+\alpha)}\|\nabla \bar{u}\|_{2}^{2}, \frac{(1-\theta) g\left(1+\epsilon_{1}\right)}{5(N+\alpha)}\|\nabla \bar{u}\|_{2}^{2}, 1, \frac{\rho \delta}{8}\right\}
$$

instead of $[26,(2.55)$ and $\varepsilon]$, respectively.

Proof of Theorem 1.1 In view of Lemma 2.7, Lemma 2.8, and Lemma 2.11, there exists $\bar{u} \in \mathcal{M}$ such that

$$
\mathcal{I}(\bar{u})=\inf _{u \in H^{1}\left(\mathbb{R}^{N}\right) \backslash\{0\}} \max _{t>0} \mathcal{I}\left(u_{t}\right)=m>0, \quad \mathcal{I}^{\prime}(\bar{u})=0 .
$$

This shows that $\bar{u}$ is a ground state solution of (1.1). 
Acknowledgements

The authors would like to thank the referees for their useful suggestions which have significantly improved the paper.

\section{Funding}

This work is supported by the National Natural Science Foundation of China (No. 11961014) and Guangxi Natural Science Foundation (2021GXNSFAA196040, 2018GXNSFAA281021).

Availability of data and materials

No data and material were used to support the work.

Code availability

No software was applied to support the work.

\section{Declarations}

\section{Competing interests}

The authors declare that they have no competing interests.

\section{Authors' contributions}

The authors declare that the study was realized in collaboration with the same responsibility. All authors read and approved the final manuscript.

\section{Publisher's Note}

Springer Nature remains neutral with regard to jurisdictional claims in published maps and institutional affiliations.

Received: 16 September 2021 Accepted: 17 November 2021 Published online: 04 December 2021

\section{References}

1. Lieb, E.H., Loss, M.: Analysis Graduate Studies in Mathematics. Am. Math. Soc., Providence (1997)

2. Pekar, S.: Untersuchungöber die Elektronentheorie der Kristalle. Akademie Verlag, Berlin (1954)

3. Moroz, I.M., Penrose, R., Tod, P.: Spherically-symmetric solutions of the Schrödinger-Newton equations. Class. Quantum Gravity 15, 2733-2742 (1998)

4. Bahrami, M., Großardt, A., Donadi, S., Bassi, A.: The Schrödinger-Newton equation and its foundations. New J. Phys. 16,115007 (2014)

5. Cingolani, S., Secchi, S., Squassina, M.: Semi-classical limit for Schrödinger equations with magnetic field and Hartree-type nonlinearities. Proc. R. Soc. Edinb. A 140, 973-1009 (2010)

6. Diósi, L.: Gravitation and quantum-mechanical localization of macro-objects. Phys. Lett. A 105, 199-202 (1984)

7. Lieb, E.H.: Existence and uniqueness of the minimizing solution of Choquard's nonlinear equation. Stud. Appl. Math. 57, 93-105 (1976)

8. Lions, P.L.: The Choquard equation and related questions. Nonlinear Anal., Theory Methods Appl. 4, 1063-1072 (1980)

9. Lions, P.L.: The concentration-compactness principle in the calculus of variations. The locally compact case. Ann. Inst. Henri Poincaré, Anal. Non Linéaire 1, 109-145 (1984)

10. Moroz, V., Van Schaftingen, J.: Groundstates of nonlinear Choquard equations: existence, qualitative properties and decay asymptotics. J. Funct. Anal. 265, 153-184 (2013)

11. Van Schaftingen, J., Xia, J.K.: Groundstates for a local nonlinear perturbation of the Choquard equations with lower critical exponent. J. Math. Anal. Appl. 464, 1184-1202 (2018)

12. Brezis, H., Nirenberg, L.: Positive solutions of nonlinear elliptic equations involving critical Sobolev exponents Commun. Pure Appl. Math. 36, 437-477 (1983)

13. Gao, F.S., Yang, M.B.: On nonlocal Choquard equations with Hardy-Littlewood-Sobolev critical exponents. J. Math Anal. Appl. 448, 1006-1041 (2017)

14. Willem, M.: Minimax Theorems Progress in Nonlinear Differential Equations and Their Applications. Birkhäuser, Boston (1996)

15. Brock, F., Solynin, A.Y: An approach to symmetrization via polarization. Trans. Am. Math. Soc. 352, 1759-1796 (2000)

16. Cassani, D., Van Schaftingen, J., Zhang, J.J.: Groundstates for Choquard type equations with Hardy-Littlewood-Sobolev lower critical exponent. Proc. R. Soc. Edinb. A 135, 1-24 (2018)

17. Tang, X.H., Chen, S.T.: Ground state solutions of Nehari-Pohožaev type for Kirchhoff-type problems with general potentials. Calc. Var. Partial Differ. Equ. 56, 1-25 (2017)

18. Qin, D.D., Tang, X.H.: On the planar Choquard equation with indefinite potential and critical exponential growth J. Differ. Equ. 285, 40-98 (2021)

19. Qin, D.D., Lai, L.Z., Yuan, S., Wu, Q.F.: Ground states and multiple solutions for Choquard-Pekar equations with indefinite potential and general nonlinearity. J. Math. Anal. Appl. 500, 125143 (2021)

20. Wu, Q.F., Qin, D.D., Chen, J.: Ground states and non-existence results for Choquard type equations with lower critical exponent and indefinite potentials. Nonlinear Anal. 197, 111863 (2020)

21. Zhang, J., Wu, Q.F., Qin, D.D.: Semiclassical solutions for Choquard equations with Berestycki-Lions type conditions. Nonlinear Anal. 188, 22-49 (2019)

22. Zhang, X.Y.: Existence and multiplicity of solutions for a class of elliptic boundary value problems. J. Math. Anal. Appl. 410, 213-226 (2014)

23. Wang, L.B., Zhang, X.Y., Fang, H.: Multiplicity of solutions for a class of quasilinear elliptic systems in Orlicz-Sobolev spaces. Taiwan. J. Math. 21, 881-912 (2017) 
24. Wang, L.B., Zhang, X.Y., Fang, H.: Existence and multiplicity of solutions for a class of $\left(\phi_{1}, \phi_{2}\right)$-Laplacian elliptic system in $\mathbb{R}^{N}$ via genus theory. Comput. Math. Appl. 72, 110-130 (2016)

25. Tang, X.H., Wei, J.Y., Chen, S.T.: Nehari-type ground state solutions for a Choquard equation with lower critical exponent and local nonlinear perturbation. Math. Methods Appl. Sci. 43, 1-12 (2020)

26. Chen, S.T., Tang, X.H.: Berestycki-Lions conditions on ground state solutions for a nonlinear Schrödinger equation with variable potentials. Adv. Nonlinear Anal. 9, 496-515 (2020)

27. Chen, S.T., Tang, X.H.: Ground state solutions for generalized quasilinear Schrödinger equations with variable potentials and Berestycki-Lions nonlinearities. J. Math. Phys. 59, 1-18 (2018)

28. Pohožaev, S.I. Eigenfunctions of the equation $\Delta u+\lambda f(u)=0$. Sov. Math. Dokl. 5, 1408-1411 (1965)

29. Li, X.F., Ma, S.W., Zhang, G.: Existence and qualitative properties of solutions for Choquard equations with a local term. Nonlinear Anal., Real World Appl. 45, 1-25 (2019)

30. Tang, X.H., Chen, S.T.: Singularly perturbed Choquard equations with nonlinearity satisfying Berestycki-Lions assumptions. Adv. Nonlinear Anal. 9, 413-437 (2020)

31. Tang, X.H., Chen, S.T.: Ground state solutions of Nehari-Pohožaev type for Schrödinger-Poisson problems with general potentials. Discrete Contin. Dyn. Syst., Ser. A 37, 4973-5002 (2017)

32. Chen, S.T., Tang, X.H.: Ground state solutions for general Choquard equations with a variable potential and a local nonlinearity. Rev. R. Acad. Cienc. Exactas Fís. Nat., Ser. A Mat. 114, 14 (2020)

\section{Submit your manuscript to a SpringerOpen ${ }^{\circ}$ journal and benefit from:}

- Convenient online submission

- Rigorous peer review

- Open access: articles freely available online

- High visibility within the field

- Retaining the copyright to your article

Submit your next manuscript at $\boldsymbol{~ s p r i n g e r o p e n . c o m ~}$ 